Supporting Information for:

\title{
Photocatalytic Reactive Oxygen Species Formation by Semiconductor- Metal Hybrid Nanoparticles. Toward Light-Induced Modulation of Biological Processes
}

Nir Waiskopf ${ }^{1,2}$, Yuval Ben-Shahar ${ }^{1}$, Michael Galchenko ${ }^{1}$, Inbal Carmel ${ }^{1}$, Gilli Moshitzky ${ }^{2}$, Hermona Soreq ${ }^{2, *}$, Uri Banin ${ }^{1 *}$

${ }^{1}$ Institute of Chemistry and the Center for Nanoscience and Nanotechnology,

${ }^{2}$ Department of Biological Chemistry and the Edmond and Lily Safra Center of Brain Science, The Hebrew University of Jerusalem, Safra Campus, Givat Ram, Jerusalem 91904, Israel Email: Uri.Banin@mail.huji.ac.il

\section{Experimental details}

Chemicals: Trioctylphosphine (TOP, Sigma Aldrich, 90\%) was vacuum distilled before use and stored under inert atmosphere. All other chemicals were used as purchased: cadmium oxide (>99.99\%), trioctylphosphine oxide (TOPO, 99\%), 1-octadecene (ODE, technical grade, 90\%), oleic acid (95\%), octadecylamine (ODA, $\geq 99 \%$ ), didodecyldimethylammonium bromide (DDAB, 98\%) and Gold(III)chloride (99\%), Gold nanoparticles (5 nm diameter stabilized suspension in citrate buffer), L-glutathione reduced (GSH, $\geq 98.0 \%$ ), Poly(styreneco-maleic anhydride), cumene terminated (PSMA), sodium sulfide nonahydrate ( $\geq 98.0 \%)$, sodium sulfite (>98\%), Polyethylenimine (PEI, branched average $\mathrm{Mw}$ 25,000), 4aminoantipyrine (4-AAP, $\geq 98.0 \%$ ), Superoxide dismutase from bovine erythrocytes (BioUltra, lyophilized powder, $\geq 4,500$ units/mg protein, $\geq 97 \%$ ), phenol $(\geq 99.5 \%$ ), terephthalic acid (TPA, 98\%), 5,5-dimethyl-1-pyrroline-N-oxide (DMPO) were purchased from Sigma Aldrich. Oleylamine (technical grade, 90\%) was purchased from Across. Octadecylphosphonic acid (ODPA) and hexylphosphonic acid (HPA) were purchased from PCI Synthesis. Sulfur (>99.0\%) was purchased from Merck.

Synthesis of CdSe seeds: $\mathrm{CdO}(0.12 \mathrm{~g}$ ), trioctylphosphine oxide (TOPO; 6.0g) and octadecylphosphonic acid (ODPA; 0.56g) were mixed in a $100 \mathrm{~mL}$ three-neck flask. The mixture was heated to $100^{\circ} \mathrm{C}$ and placed under vacuum for 1 hour followed by three times of argon purging. Under argon atmosphere, the solution was heated to $300^{\circ} \mathrm{C}$ to dissolve the $\mathrm{CdO}$, forming a clear colorless solution. At this temperature, $1.8 \mathrm{~mL}$ of TOP was injected into the hot solution. Next, the solution was further heated to $350^{\circ} \mathrm{C}$, at which $0.87 \mathrm{~mL}$ of precursor solution of selenium $(0.21 \mathrm{~g})$ in TOP $(1.7 \mathrm{~mL})$, was rapidly injected into the hot solution. The 
reaction time was, typically, $25 \mathrm{sec}$ for CdSe seeds with diameter of $\sim 2.3 \mathrm{~nm}$. The reaction was quenched by removing the heating mantle and cooling with fan. The crude reaction mixture was diluted with toluene. Methanol was added in order to precipitate the nanocrystals and remove excess surfactants.

Synthesis of seeded CdSe/CdS nanorods: CdO (0.065g), trioctylphosphine oxide (TOPO; $3.0 \mathrm{~g}$ ), octadecylphosphonic acid (ODPA; 0.29g) and hexylphosphonic acid (HPA; 0.065g) were mixed in a $100 \mathrm{~mL}$ three-neck flask. The mixture was heated to $100^{\circ} \mathrm{C}$ and placed under vacuum for 1 hour followed by three times of argon purging. Under argon atmosphere, the solution was heated to $300^{\circ} \mathrm{C}$ and at this temperature $1.8 \mathrm{~mL}$ TOP was injected into the hot solution. Next, the solution was further heated to $360^{\circ} \mathrm{C}$, at which a precursor solution of CdSe seeds (typically, $4 \times 10^{-8} \mathrm{~mol}$ ) with sulfur in TOP solution $(1.6 \mathrm{~mL} ; 0.075 \mathrm{~g} / \mathrm{mL})$ was rapidly injected. The temperature decreased and then recovered within 1-2min. The reaction time was, typically, $12 \mathrm{~min}$. The reaction was quenched by removing the heating mantle and cooling with fan. The crude reaction mixture was diluted with toluene. Methanol was added in order to precipitate the nanocrystals and remove excess surfactants. Absorption spectra of both $\mathrm{CdSe}$ seeds and $\mathrm{CdSe} / \mathrm{CdS}$ nanorods (NRs) are presented in Supplementary Figure S1 including the CdSe seeds feature within the seeded rod structure (inset).

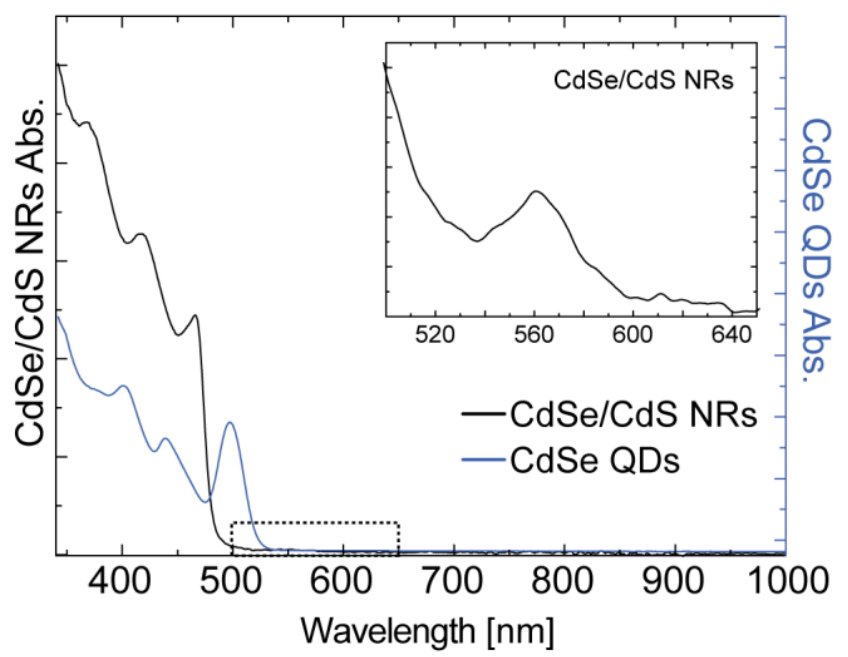

Supplementary Figure S1. Absorption spectra of CdSe QDs (blue) and CdSe/CdS NRs (black). Inset showing the CdSe seed feature within the seeded rod structure.

Synthesis of CdS seeds: CdS nanocrystal seeds were synthesized by a modification of a previously reported procedure. ${ }^{1}$ Cadmium oxide $(\mathrm{CdO} ; 0.106 \mathrm{~g})$, oleic acid $(\mathrm{OA} ; 2.26 \mathrm{~g})$ and 1 octadecene (ODE; 20g) were mixed in a $100 \mathrm{~mL}$ three-neck flask. The mixture was heated to $100^{\circ} \mathrm{C}$ and placed under vacuum for 1 hour followed by three times of argon purging. Under 
argon atmosphere, the solution was heated to $260^{\circ} \mathrm{C}$ to dissolve the $\mathrm{CdO}$, forming a clear colorless solution. A precursor solution consisting of sulfur $(0.013 \mathrm{~g})$ and ODE $(7 \mathrm{~mL})$ was rapidly injected into the hot solution. The reaction time was typically $90 \mathrm{sec}$ for $\mathrm{CdS}$ seeds with diameter of $\sim 3.1 \mathrm{~nm}$. The reaction was quenched by removing the heating mantle and cooling with fan. The crude reaction mixture was precipitated with acetone followed by centrifugation. For further purification, the particles were dissolved in toluene and the precipitation procedure was repeated several times.

Synthesis of CdS nanorods: CdS nanorods were synthesized by a modification of a previously reported procedure employing seeded growth. ${ }^{2} \mathrm{CdO}(0.12 \mathrm{~g})$, trioctylphosphine oxide (TOPO; 6.0g), octadecylphosphonic acid (ODPA; 0.68g) and hexylphosphonic acid (HPA; $0.04 \mathrm{~g}$ ) were mixed in a $100 \mathrm{~mL}$ three-neck flask. The mixture was heated to $100^{\circ} \mathrm{C}$ and placed under vacuum for 1 hour followed by three times of argon purging. Under argon atmosphere, the solution was heated to $350^{\circ} \mathrm{C}$ and at this temperature trioctylphosphine (TOP; $1.8 \mathrm{~mL}$ ) was injected into the hot solution. Next, the solution was further heated to $365^{\circ} \mathrm{C}$, at which a precursor solution of CdS seeds (typically, $3 \times 10^{-8} \mathrm{~mol}$ ) and sulfur in TOP solution $(1.6 \mathrm{~mL} ; 0.075 \mathrm{~g} / \mathrm{mL})$ was rapidly injected into the hot solution. The temperature decreased and then recovered within $1-2 \mathrm{~min}$. The reaction time was $9 \mathrm{~min}$ for $49 \mathrm{~nm} \times 4.2 \mathrm{~nm}$ CdS rods. The reaction was quenched by removing the heating mantle and cooling with fan. The crude reaction mixture was diluted with toluene. Methanol was added in order to precipitate the nanocrystals and remove excess surfactants.

Synthesis of CdS-Au and CdSe/CdS-Au hybrid nanorods: A precursor stock solution consisting of octadecylamine (ODA; $0.055 \mathrm{~g}$ ), didodecylammonium bromide (DDAB; $0.021 \mathrm{~g}$ ) and $\mathrm{AuCl}_{3}(0.010 \mathrm{~g})$ in toluene $(10 \mathrm{~mL})$ was sonicated for $15 \mathrm{~min}$ to dissolve the $\mathrm{AuCl}_{3}$, and the solution changes color from dark brown to yellow. In order to achieve selective growth of 1.5$1.8 \mathrm{~nm}$ gold tips on one apex of the NRs, a molar ratio of 700-900 Au ions per NR was used depending on the specific properties of the rods. Diluted Au growth stock solution was added to NRs (typically $\sim 2 \times 10^{-9} \mathrm{~mol}$ ) in toluene $(20 \mathrm{~mL})$ in $100 \mathrm{~mL}$ flask under flowing argon. The solutions are mixed for 1 hour at room temperature and under dark conditions. The product hybrid nanoparticles (HNPs) are then washed and precipitated with acetone followed by separation via centrifugation. ${ }^{3}$ The hybrid samples had narrow size distribution according to their absorption spectra and TEM characterization measurements and statistics (Supplementary Figure S2). 
Nanoparticle Characterization: TEM characterization was performed using a Tecnai T12 G2 Spirit and Tecnai F20 G2. All size statistics are done with "Scion image" program on 200 particles. Absorption was measured with a JASCO V-570 UV-vis-near IR spectrophotometer. Extinction coefficient values of the NRs were calculated using a previously reported method. ${ }^{4}$
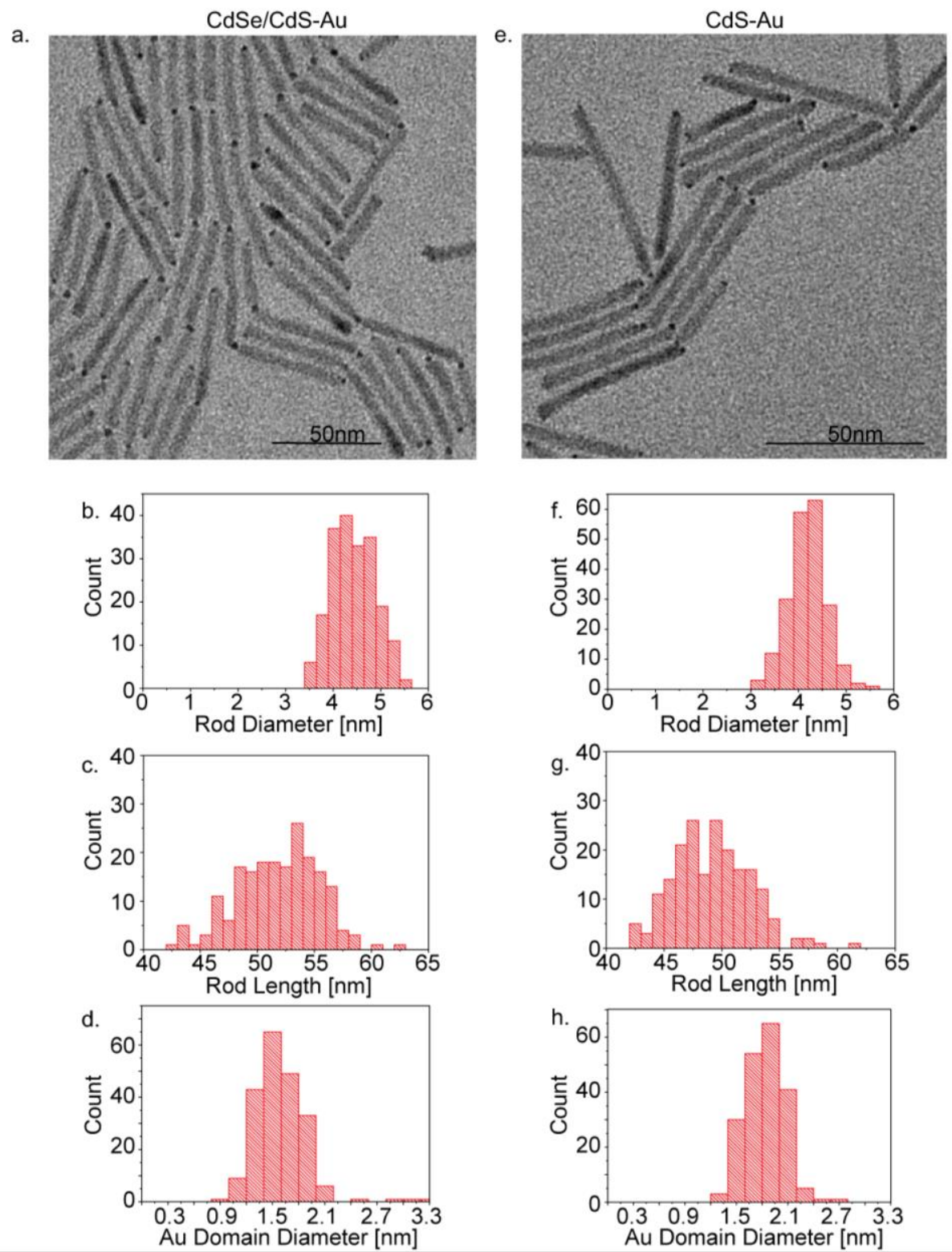

Supplementary Figure S2. TEM image of hybrid nanorods CdSe/CdS-Au $(52 \mathrm{~nm} \times 4.4 \mathrm{~nm})$ (a) and CdS-Au (49nm x 4.2nm) (e). Sizing histograms for rods diameter (b,f), length (c,g) and Au metal domain diameter $(\mathrm{d}, \mathrm{h})$.

Phase transfer: NPs were transferred to water by ligand exchange and polymer coating methods. For exchanging the native organic-soluble ligands with the thiolate alkyl ligands, the ligand exchange strategy was used. Stock solution of L-glutathione (GSH) was prepared by dissolving GSH (140mg) and $\mathrm{KOH}(100 \mathrm{mg})$ in methanol $(1 \mathrm{~mL})$. Next, $200 \mu \mathrm{L}$ of stock 
solution is added to NPs in chloroform $(1 \mathrm{~mL})$ with an optical density of 1.5 at the CdS first state transition and mixed for 1-2 min. Based TDW (pH 11-12) is added to the flocculated solution and after mixing phase separation appears and the NPs are extracted from the upper water phase after mild centrifugation.

Polymer coating was done with different polymers. ${ }^{5,6}$ Poly(styrene-co-maleic anhydride) (PSMA) coating is achieved by mixing $2 \mathrm{~mL}$ of nanoparticles solution with PSMA $(20 \mathrm{mg})$ in chloroform $(1 \mathrm{~mL})$ for 5 hours. Then ethanolamine $(20 \mu \mathrm{L})$ is added to the solution and mixed for 1-2 min. Next, recurring additions of TDW $(1 \mathrm{~mL})$ is done to transfer the particles to the above water phase followed by mild centrifugation before extraction. Polymer coating with polyethylenimine (PEI) was done by mixing NPs solution $(1 \mathrm{~mL})$ with PEI $(0.15 \mathrm{~g}$; MW $25,000)$ in chloroform $(1 \mathrm{~mL})$ for 1 hour. Then the particles are precipitated and washed with cyclohexane (1:1 chloroform/cyclohexane), followed by centrifugation. TDW is added to the precipitate and residues of PEI are removed by centrifugation.

Before use all nanoparticle solutions were washed through 100KDa cellulose membrane, to remove excess of polymer and ligands.

HRP activity assay: The catalytic activity of the enzyme was measured spectroscopically following the change in the absorption spectrum due to the production of quinoneimine dye by HRP, as illustrated in Supplementary Figure S3a. ${ }^{7}$ Typically, $20 \mu \mathrm{L}(1 \mathrm{mg} / 2.5 \mathrm{~mL}) \mathrm{HRP}$ and $25 \mu \mathrm{L} \quad(1 \mathrm{mg} / \mathrm{mL}) \quad \mathrm{SOD}, \quad 200 \mu \mathrm{L} \quad(8.125 \mathrm{mg} / 1 \mathrm{~mL})$ 4-aminoantipyrine (4-AAP), $600 \mu \mathrm{L}$ $(79 \mathrm{mg} / \mathrm{mL})$ phenol and $100 \mu \mathrm{L}$ of $10-100 \mathrm{nM}$ HNPs/NPs were dissolved and mixed with PBS (final volume $2 \mathrm{~mL}$ ). Then samples were irradiated using $405 \mathrm{~nm}$ laser $\left(20 \mathrm{~mW} / \mathrm{cm}^{2}\right)$ while measuring absorption.

Control experiments for the photo-switched HRP activation: Control measurements under various conditions were done in order to isolate the light induced HRP activation by NPs excitation from the intrinsic enzyme activity, or non-specific effects of the light excitation. As shown in Supplementary Figure S3b, inert atmosphere e.g. absence of oxygen (red curve), absence of HNPs (green curve) or without irradiation (pink curve), all show negligible activity and product formation in comparison to the product formation under the presence of the formers (black curve). This confirms their key role in the formation of the product. Unspecific product formation was also measured in the absence of enzyme (blue curve). This falsepositive signal could be attributed to phenol oxidation by presence of ROS following light irradiation. Note that the phenol oxidation is not mediated through direct charge transfer from the nanoparticles given the absence of this response under inert conditions. Therefore, in order 
to obtain the effective net contribution of the HNPs to the HRP activation, we subtracted this signal from all data and figures, presented in this frame of work.

a.

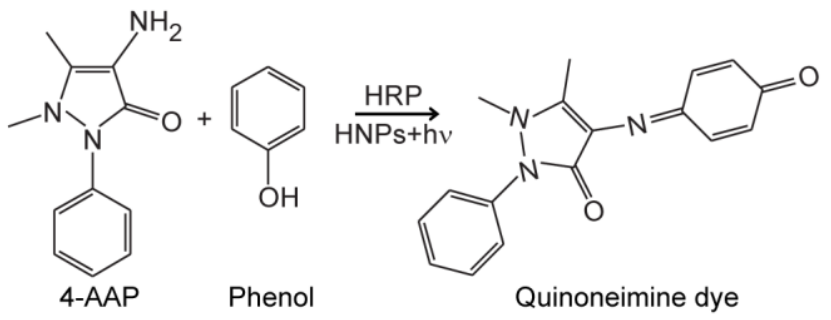

b.

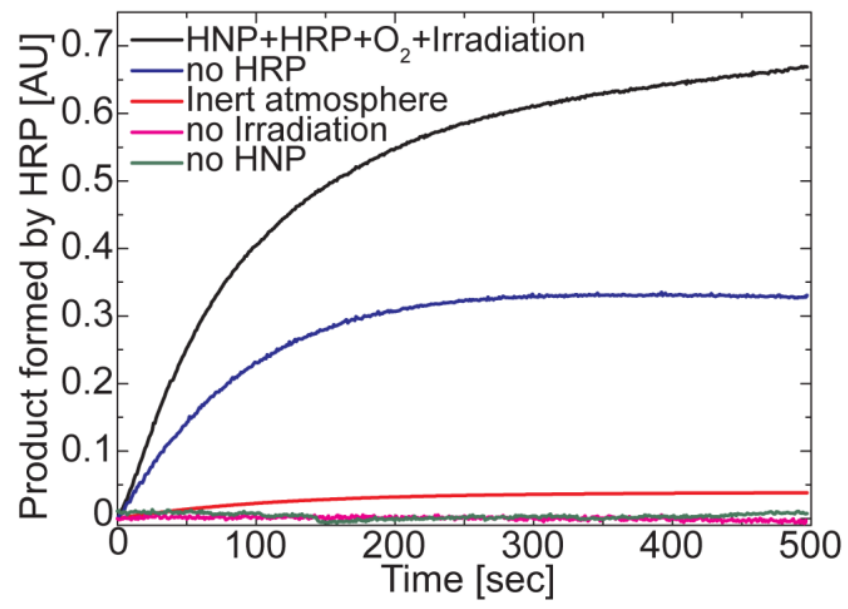

Supplementary Figure S3. (a) Reaction scheme for production of quinoneimine catalyzed by HRP in the presence of 4-AAP and phenol upon light stimulation of HNPs. (b) Control measurements of photo-induced modulation of HRP activity by HNPs were done in various conditions, absence of enzyme (blue curve) or oxygen (red curve), without irradiation (pink curve) and absence of HNPs (green curve). The result of the full assay which includes all these parameters is shown in the black curve.

CdS-Au HNPs show higher light induced HRP activation than CdS NRs: The photoinduced activity of HRP was also investigated with CdS based nanosystem as photocatalysts. Comparison between CdS-Au HNPs and CdS NRs showed similar trends to those reported in the main manuscript for CdSe/CdS based nanosystem. As shown in Supplementary Figure S4, in all conditions, native and in the presence of SOD and hole scavenger (e.g. EtOH), the hybrid system (black and red curves) reveals higher catalytic function and efficiency than the semiconductor alone (blue and orange curves). In addition, the effects of the hole scavenger and the presence of SOD enzyme on the overall HRP activity are repeated in this system as was demonstrated for the $\mathrm{CdSe} / \mathrm{CdS}$ based systems. The contribution of each of the additives, $\mathrm{SOD}$ and $\mathrm{EtOH}$ is discussed in detail within the main manuscript. 


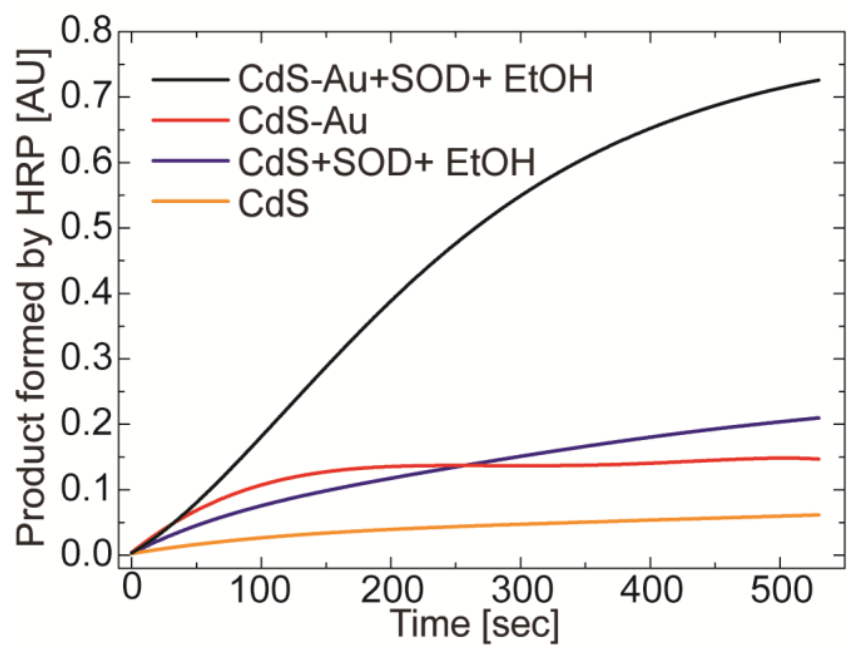

Supplementary Figure S4. Comparison of HRP activity upon light irradiation between CdSAu HNPs and CdS NRs in the presence of SOD and hole scavenger (e.g. EtOH) (black and blue curves, respectively) and in the absence of these additives (red and orange curves, respectively).

Hydroxyl radical detection: TPA in PBS $(1340 \mu \mathrm{L}, 0.236 \mathrm{mg} / \mathrm{mL})$ was mixed with NPs $(100 \mu \mathrm{L}, \sim 60 \mathrm{nM})$ and PBS was added for a total volume of $2 \mathrm{~mL}$. Samples were irradiated using $405 \mathrm{~nm}$ laser $\left(20 \mathrm{~mW} / \mathrm{cm}^{2}\right)$ and emission was measured with a Cary Eclipse Fluorometer (Varian Inc.), every minute after excitation at $310 \pm 5 \mathrm{~nm}$.

Electron paramagnetic resonance (EPR): ROS were detected by EPR spin-trapping technique coupled with 5,5-Dimethyl-1-pyrroline N-oxide (DMPO) as the spin trap molecule. DMPO was purified in double distilled water, with activated charcoal in the dark. After 1530 minutes, the DMPO solution was filtered and its concentration was determined spectrophotometrically, using $\varepsilon_{277 \mathrm{~nm}}=8000 \mathrm{M}^{-1} \mathrm{~cm}^{-1}$. ${ }^{8}$ The solution was stored at $20^{\circ} \mathrm{C}$ for no longer than 2 weeks. Samples containing aqueous suspensions of nanoparticles and DMPO $(10 \mathrm{mM})$ were drawn by a syringe into a gas-permeable Teflon capillary (Zeus Industries, Raritan, NJ) and inserted into a narrow quartz tube that was open at both ends. Then, the tube was placed into the EPR cavity (ER 4102ST) and spectra were recorded, using Bruker EPR 100d X-band spectrometer, during or after illumination with $405 \mathrm{~nm} 20 \mathrm{~mW} / \mathrm{cm}^{2}$ laser. The EPR measurement conditions were as follows: frequency: $9.77 \mathrm{GHz}$; microwave power: 20mW; scan width: $65 \mathrm{G}$; center field: $3458 \mathrm{G}$; resolution: 1024 ; receiver gain: $1 \times 105$; conversion time: $82 \mathrm{msec}$; time constant: $328 \mathrm{msec}$; sweep time: $84 \mathrm{sec}$; \# scans=2; modulation amplitude: 2G; After acquisition, simulations of the recorded spectra were performed using an algorithm provided in the WINSIM program, which is available from NIEHS (National 
Institutes of Health, web site: http:// epr.niehs.nih.gov/pest_mans/winsim.html). The simulation parameters used for analysis with comparison to relevant parameters reported from the literature are summarized in the following Supplementary Table1.

Supplementary Table S1. Simulation parameters used for EPR measurements (DMPO radical trapping agent) with comparison to relevant literature parameters ${ }^{9}$ :

\begin{tabular}{|c|c|c|c|}
\hline \multicolumn{4}{|l|}{ Simulation } \\
\hline Radicals & $\mathbf{A}_{\mathbf{N}} / \mathbf{G}$ & $\mathbf{A}_{\mathbf{H}} / \mathbf{G}$ & Additional $A_{H} / G$ \\
\hline$\cdot \mathrm{OH}$ & 15 & 15 & \\
\hline$\cdot \mathrm{OOH}$ & 14.1 & 11.3 & 1.25 \\
\hline $\mathrm{CH}_{3} \mathrm{C}^{*} \mathrm{HOH}$ & 15.8 & 22.8 & \\
\hline $\mathrm{SO}_{3}{ }^{-}$ & 14.4 & 15.9 & \\
\hline Unspecific signal & 14.5 & & \\
\hline \multicolumn{4}{|l|}{ Literature } \\
\hline Radicals & $A_{N} / G$ & $\overline{A_{H} / G}$ & Additional $\mathbf{A}_{\mathbf{H}} / \mathbf{G}$ \\
\hline$\cdot \mathrm{OH}$ & $14.77-15.2$ & $14.77-15.2$ & \\
\hline$\cdot \mathrm{OOH}$ & $14.1-14.3$ & $11.3-11.7$ & 1.25 \\
\hline $\mathrm{CH}_{3} \mathrm{C}^{*} \mathrm{HOH}$ & 15.8 & 22.8 & \\
\hline $\mathrm{SO}_{3}{ }^{-}$ & $14.4-14.7$ & $15.9-16.5$ & \\
\hline
\end{tabular}

A time scan mode was used to follow on the increase in signal intensity upon in-situ illumination. The time measurements were taken at static magnetic field of $3448 \mathrm{G}$, which fits the maximum of the second group of peaks from the low field, attributed to the DMPO adduct of superoxide radicals (DMPO-OOH). The parameters were similar to the field sweep mode except: conversion time: 328msec; time constant: 328msec; sweep time: 671sec; \# scans $=1$. 
Similar EPR experiments as were presented in the manuscript were also done in the presence of ethanol which can scavenge hydroxyl radicals (Supplementary Figure S5).

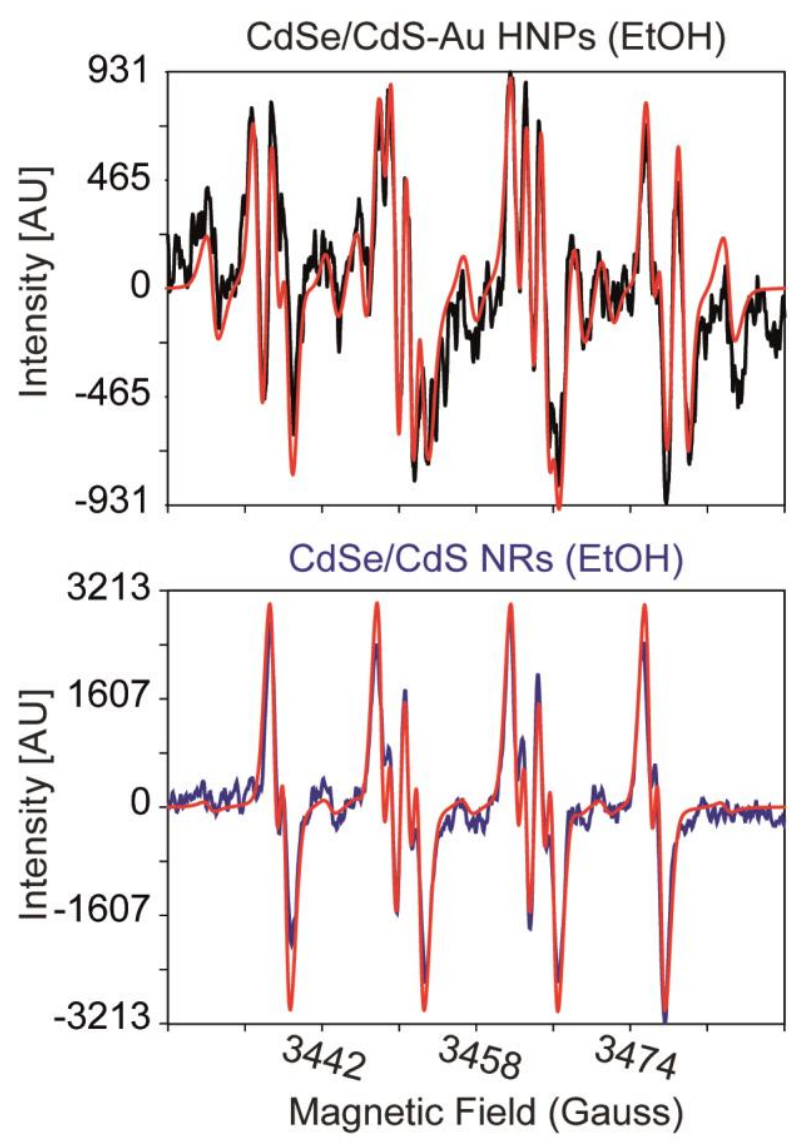

Supplementary Figure S5. EPR measurements during illumination of HNPs or NRs (upper and lower panels, respectively) in the presence of ethanol resulted in decreased signal of DMPO-OH and in increase in signal with hyperfine coupling of $a_{N}=15.8 G$ and $a_{H}=22.8 G$ which indicates the presence of $\mathrm{CH}_{3} \mathrm{C}^{*} \mathrm{HOH}$, an ethanol-derived radical adduct. This confirms the DMPO-OH results from the presence of hydroxyl radicals in the solution.

Oxygen consumption measurements: Oxygen consumption was measured by polarography with a thermostatically controlled $\left(37^{\circ} \mathrm{C}\right)$ Clark oxygen electrode (Strathkelvin 782 Oxygen System; Strathkelvin Instrument Ltd.). ${ }^{10} \mathrm{HNPs} / \mathrm{NRs}$ with PEI and GSH surface coating with the typical concentrations of the HRP activity assay $(100 \mu \mathrm{L}$ of $10-100 \mathrm{nM})$ in PBS buffer solution were stirred in a closed cell. Under irradiation $\left(405 \mathrm{~nm}\right.$ laser $20 \mathrm{~mW} / \mathrm{cm}^{2}$ ) oxygen consumption was recorded for typically $10 \mathrm{~min}$ and calculated as rate of change in the oxygen concentration. 
Glutathione as an alternative surface coating: As described in the phase transfer section above, both hybrids and semiconductor NPs were transferred to aqueous solutions with additional ligands including GSH via ligand exchange mechanism. The GSH coated systems were investigated for the photo-switched HRP activity under the same conditions as for PEI coated systems reported within the main manuscript. Similar behavior was observed for the GSH coated systems with regard to the trends shown for PEI surface coating.
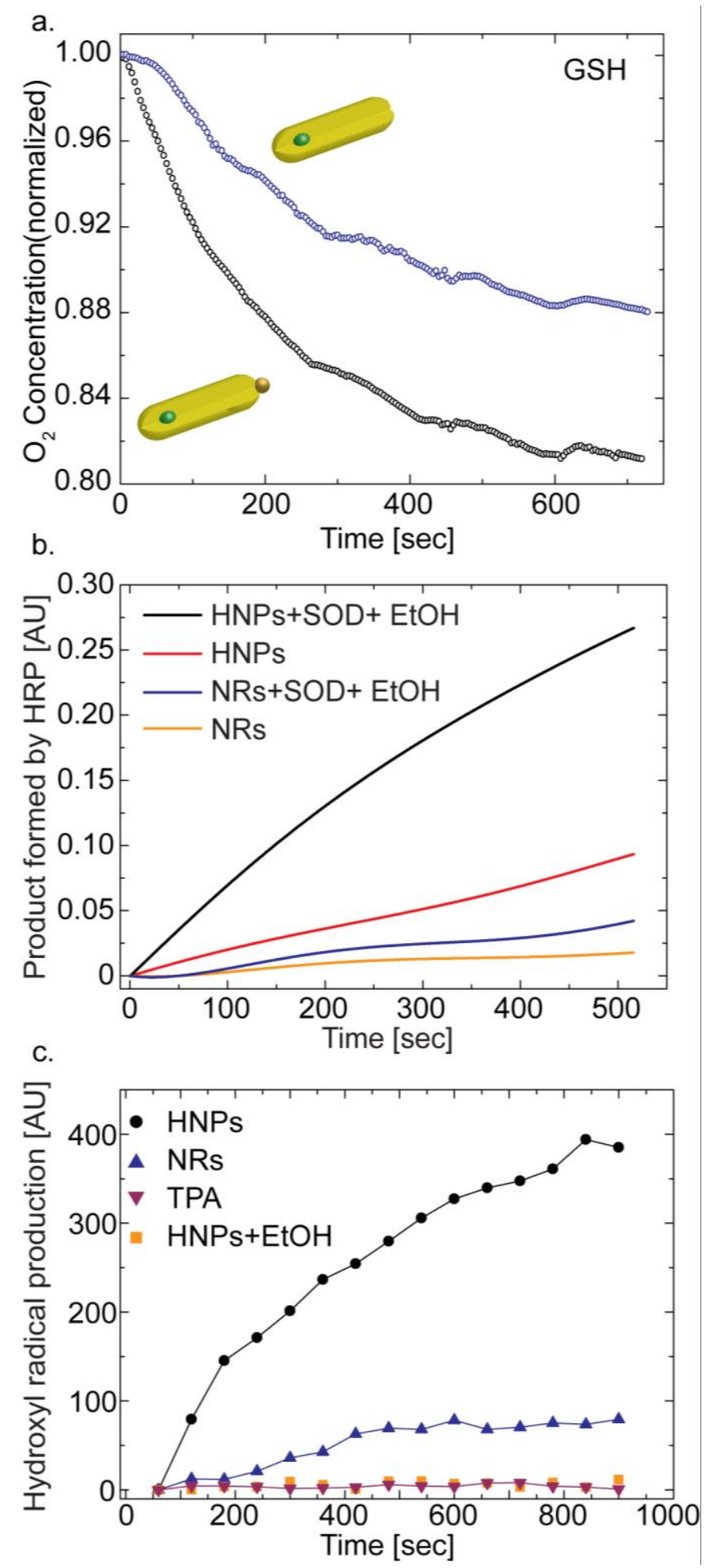
Supplementary Figure S6. (a) Kinetic measurements of molecular oxygen consumption by HNPs and NRs coated with GSH upon light illumination, measured by polarography. (b) Comparison of HRP activity upon light irradiation using the HRP activity assay between $\mathrm{CdSe} / \mathrm{CdS}-\mathrm{Au}$ HNPs and CdSe/CdS NRs coated by GSH in the presence of SOD and hole scavenger (e.g. EtOH) (black and blue curves, respectively) and in the absence of these additives (red and orange curves, respectively). (c) Kinetic measurements of hydroxyl radical formation using the TPA assay showing faster hydroxyl radical production upon stimulation of hybrids (black) in comparison to bare nanorods (blue). Addition of ethanol as a hole scavenger to the hybrids solution (orange) prevents this reaction, yielding similar signals to those of control TPA alone (purple).

\section{Kinetic photocatalytic activity measurements for HNPs with different biological hole} acceptors: In order to determine the photocatalytic activity of HNPs in the presence of different biological hole acceptors, hydrogen gas generation via the photocatalytic water reduction reaction was measured. The photocatalysts were dispersed in PBS solution $(2 \mathrm{~mL}$; optical density, OD $\sim 1$ at $405 \mathrm{~nm}$ ). The photocatalyst solution was placed in a quartz cuvette and hole scavengers (typically $0.05 \mathrm{M}$ ), were added to the solution. The solution is purged with argon for 20min and stirred to achieve oxygen free condition in which ROS formation is suppressed and hydrogen formation occurs. The HNPs were then illuminated with $405 \mathrm{~nm}$ $28 \mathrm{~mW} / \mathrm{cm}^{2}$ laser, producing $5.7 \times 10^{16}$ photons/sec. Aliquots of the reaction vessel head space were taken at different time intervals and the hydrogen was detected and quantified using

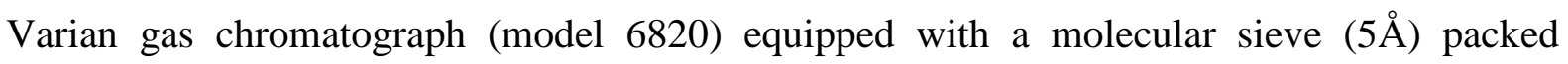
column and a thermal conductivity detector. The resulting chromatograms and hydrogen concentration are obtained by the comparison to a calibration curve of known hydrogen amounts.

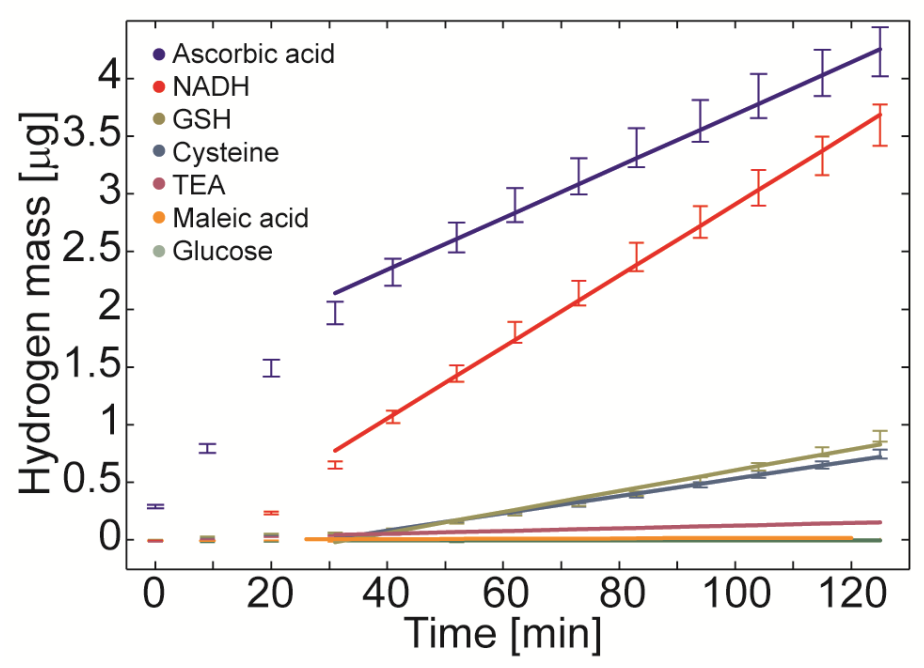


Supplementary Figure S7. Kinetic measurements of hydrogen production with different biological molecules as hole acceptor agents in presence of CdS-Au HNPs (PEI coated).

Cell Culture: K-562 human bone marrow cell line (ATCC ${ }^{\circledR}$ CCL-243 ${ }^{\mathrm{TM}}$ ) were grown in $5 \% \mathrm{CO}_{2}, 37^{\circ} \mathrm{C}$ incubator in medium consisting of RPMI 1640 (Sigma, Cat\# R0883) supplemented with $10 \%$ heat inactivated fetal bovine serum (Biological industries, Cat\# 04121-1A), 1\% L-glutamine (Biological industries, Cat\# 03-020-1A) and 1\% penicillin and streptomycin (Gibco, Cat\# 15140-122). Cells were split every 3-4 days.

Biocompatibility: The cytotoxic effects of the HNPs were assessed using Live/Dead assay and MTT viability test with K-562 cell line. Typically, cells were incubated with $10 \mu \mathrm{L}$ of colloidal HNPs and CdSe/CdS nanorods solutions (1-100nM) and $190 \mu \mathrm{L}$ of cell medium in 96 well-plate. $21 \mathrm{hr}$ after MTT was added to each well $(0.25 \mathrm{mg} / \mathrm{mL}$ final concentration). Three hours after that, the cells were extracted by centrifugation at 3000RPM for 5min, cell's medium was replaced with DMSO and absorption was measured at $535 \mathrm{~nm}$ and $635 \mathrm{~nm} .{ }^{11}$ The Live/Dead assay was performed $24 \mathrm{hr}$ following the incubation with the NPs according to manufacturer instructions (molecular probes, Cat\# L-7013). Working solution was made by diluting the Live/Dead dyes in HBSS buffer. The dye solution was added to the cell pellet followed by 15 minutes incubation in the dark. The cells were incubated for 15 minutes in $4 \%$ glutaraldehyde and then observed under fluorescent microscope. Similar Live/Dead assay was done $24 \mathrm{hr}$ following illumination for $5 \mathrm{~min}$ with $405 \mathrm{~nm} 20 \mathrm{~mW} / \mathrm{cm}^{2}$ LED on the cells after their incubation for 1 hour with NRs or HNPs.
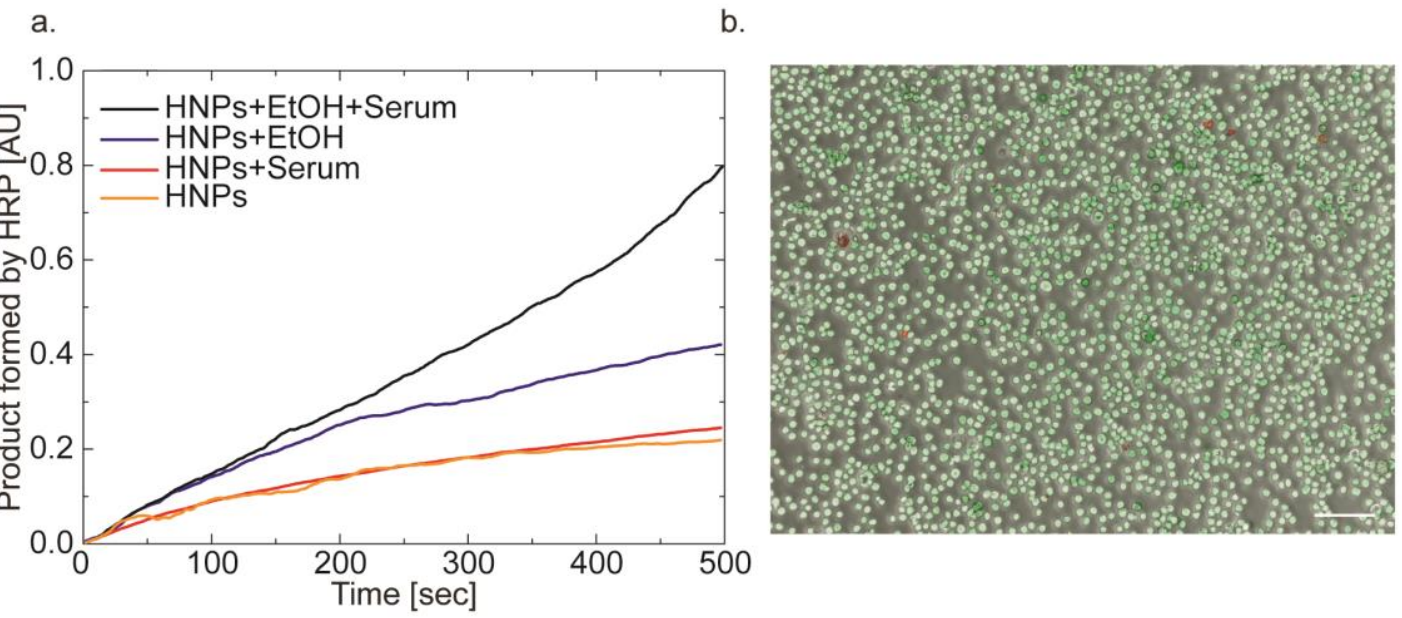

Supplementary Figure S8. (a) The production of HRP's product after stimulating HNPs in serum (black and red) or in buffer (blue and orange) with or without addition of hole acceptor to the solution. The results demonstrate the ability to use HNPs stimulation with and without 
hole acceptor for efficient and controlled production of hydrogen peroxide also in biological systems. (b) Live/Dead assay confirms incubation of K-562 cells with HNPs for $24 \mathrm{hr}$ under dark conditions didn't affect their viability (most cells are stained in green), Scale bar is $100 \mu \mathrm{m}$.

\section{References}

1. Yu, W. W.; Peng, X. G. Angew. Chem. Int. Ed. 2002, 41, 2368-2371.

2. Carbone, L.; Nobile, C.; De Giorgi, M.; Sala, F. D.; Morello, G.; Pompa, P.; Hytch, M.; Snoeck, E.; Fiore, A.; Franchini, I. R.; Nadasan, M.; Silvestre, A. F.; Chiodo, L.; Kudera, S.; Cingolani, R.; Krahne, R.; Manna, L. Nano Lett. 2007, 7, 2942-2950.

3. Menagen, G.; Mocatta, D.; Salant, A.; Popov, I.; Dorfs, D.; Banin, U. Chem. Mater. 2008, 20, 6900-6902.

4. Shaviv, E.; Salant, A.; Banin, U. ChemPhysChem 2009, 10, 1028-1031.

5. Nann, T. Chem. Commun. 2005, 1735-1736.

6. Lees, E. E.; Nguyen, T. L.; Clayton, A. H. A.; Mulvaney, P.; Muir, B. W. ACS Nano 2009, $3,1121-1128$.

7. Nicell, J. A.; Wright, H. Enzyme Microb. Tech. 1997, 21, 302-310.

8. Kalyanaraman, B. Rev. Biochem. Toxicol. 1982, 4, 73-139.

9. Buettner, G. R. Free Radical Bio. Med. 1987, 3, 259-303.

10. Brenner-Lavie, H.; Klein, E.; Ben-Shachar, D. Biochem. Pharmacol. 2009, 78, 85-95.

11. Mosmann, T. J. Immunol. Methods 1983, 65, 55-63. 